\title{
A Novel and Functionally Diverse Class of Acetylcholine-gated Ion
}

\section{Channels}

Iris Hardege ${ }^{* 1}$, Julia Morud ${ }^{* 1}$, William R Schafer ${ }^{1,2}$

\section{Affiliations}

${ }^{1}$ MRC Laboratory of Molecular Biology, Francis Crick Avenue, Cambridge, CB2 0QH, United

Kingdom

${ }^{2}$ Department of Biology, KU Leuven, Leuven, Belgium

* These authors contributed equally to this work

Corresponding Author: William R Schafer,wschafer@mrc-lmb.cam.ac.uk 


\section{Abstract}

\section{Keywords}

Choline / C.elegans / Co-expression / Polymodal / Synaptic localisation

\section{Introduction}

Rapid signalling through neuronal networks is essential for producing coordinated

22 behaviours in animals. At the fundamental level, fast neurotransmission is mediated through neurotransmitter release, resulting in activation of ion channels on the postsynaptic neuron. In 
neurotransmitters, glutamate, and acetylcholine, and two inhibitory neurotransmitters, GABA

the remaining neurotransmitters activate pentameric receptors from the Cys-loop receptor superfamily of ligand-gated ion channels (LGICs). Although LGICs are highly conserved across phyla, ligand binding properties and ion selectivity diverge significantly, resulting in a large diversity of mechanisms by which small molecules acting via LGICs can influence the activity in neuronal circuits.

Despite their conserved general structure, Cys-loop LGICs vary significantly in their

functional properties, particularly when channels from invertebrate phyla are considered. For

ivermectin (Cully et al, 1996, 1994). Many animals including insects, nematodes and mammals

also express LGICs that can be gated by aminergic ligands, including histamine-gated chloride

channels in Drosophila (Gisselmann et al, 2002), various nematode channels gated by

excitatory mammalian 5- $\mathrm{HT}_{3}$ receptors (Kondo et al, 2014; Lombaert et al, 2018). Even more

divergent roles for LGICs have been identified in marine species, where LGICs gated by terpenes and chloroquine function as chemoreceptors in octopus (van Giesen et al, 2020). nicotinic receptors and their paralogues (including the 5HT-3 receptors), and the other containing channels more closely related to $\mathrm{GABA}_{\mathrm{A}}$ receptors (Jones \& Sattelle, 2008). The $C$. elegans genome contains several subfamilies which appear to have diversified independently 
specific LGIC subfamilies. The $C$. elegans genome contains a number of $\mathrm{GABA}_{\mathrm{A}}$-like et al, 2000; Jobson et al, 2015; Ringstad et al, 2009; Putrenko et al, 2005; Margie et al, 2013;

53 Yassin et al, 2001). One of these subgroups consists of genes for monoamine-gated channels

54 (both anion and cation selective), and a second subgroup consists of eight channels, four of which are acetylcholine-gated anion channels and four that are still uncharacterized. A third subfamily, known as the "diverse group" (Hobert, 2013), is largely uncharacterised; though one member, LGC-40, has been reported to be a low affinity serotonin-gated channel also gated by acetylcholine and choline (Ringstad et al, 2009), the properties of the remaining eight channels in this subgroup, including their ligands, ion selectivity, and expression patterns, are currently unknown.

Here we describe the deorphanisation and characterisation of five new C. elegans

LGICs activated by acetylcholine, including four members of the diverse subgroup. One of these, LGC-39, forms a polymodal homomeric anion channel activated by acetylcholine as

Using public single cell RNAseq expression data together with our new electrophysiological data, we also predict the polarity of synapses in the worm connectome, as well as intracellular localisation patterns for uncharacterized LGICs. These results highlight the unexpected functional diversity of cholinergic signalling in the $C$. elegans nervous system.

\section{Results}

\section{Deorphanisation of Uncharacterised LGICs Reveals Diversity of Cholinergic Channels}


diverse group, consisting of the GGR-1 (here renamed to LGC-57) and the LGC-45 are largely unknown. To investigate the properties of these channels, we first expressed each channel gene in Xenopus oocytes and tested for current activation during the perfusion of a panel of neurotransmitters. Despite their homology to vertebrate $\mathrm{GABA}_{\mathrm{A}}$ and glycine receptors (the source of the name GGR-1), we observed no activation of any members of the GGR-1 channels LGC-57 (aka GGR-1), LGC-58 (aka GGR-2) and LGC-40 that were specifically gated by choline and acetylcholine (Figure 1A). All three channels showed a preference for choline, with $\mathrm{EC}_{50}$ values 2.5 to 3-fold lower for choline than acetylcholine (Figure 1C). These findings parallel a previous report that LGC-40 forms a choline and acetylcholine-gated channel, although in contrast to that report we did not observe serotonin responses (Ringstad et al, 2009). We did not observe currents in response to any of the tested compounds for the remaining members of the LGC-57 group (LGC-41 and LGC-42) nor for any members of the LGC-45 group (Figure S1A).

In addition to the LGC-57 and LGC-45 groups, many other $C$. elegans LGICs lack identified ligands. While several members of the so-called ACC group of LGICs have been shown to form acetylcholine-gated chloride channels, four members of this subfamily (acc-4, $\lg c-47, \lg c-48$, and $\lg c-49$ ) had not previously been characterised (Figure 1B). Upon expression in Xenopus oocytes, we found that one of these channels, LGC-49, formed an acetylcholine gated channel with an $\mathrm{EC}_{50}$ of $19 \mu \mathrm{M}$ (Figure 1C). Unlike the members of the LGC-57 group, LGC-49 showed no significant activation by choline. No ligand-induced currents were observed for ACC-4, LGC-47 or LGC-48, either alone, or in combination with closely related 
LGC-49. For all channels we observed significant reversal potential shifts following $a l, 2017)$, which to date lacked ion selectivity data; this likewise showed reversal shifts characteristic of an anion selective channel (Figure 2A-B). Interestingly, all members of the diverge from the PAR motif, both LGC-49 and LGC-46 contain the PAR motif sequence. Thus, the PAR motif appears to correlate with anion selectivity in both the LGC-57 and ACC groups of nematode acetylcholine-gated LGICs.

\section{Cholinergic Channels Display Diverse Antagonist Binding Properties}

In order to understand if there are further functional differences between the channels deorphanised in this study, we exposed each channel to three cholinergic antagonists, mecamylamine, strychnine and d-tubocurarine. Strychnine and d-tubocurarine have been mechanisms vary between LGICs of different classes (Brams et al, 2011); in contrast, mecamylamine has been shown to interact with the transmembrane regions of mammalian nAChRs (Bondarenko et al, 2014). We saw that the antagonistic profile differed significantly 
125

126

127

128

129

130

131

132

133

smallest antagonists, mecamylamine and strychnine, had a similar $\mathrm{IC}_{50}$ values for LGC-57, LGC-58a and LGC-40 (Figure 2D). However, tubocurarine, the largest molecule of the antagonists, displayed an 11-fold shift in $\mathrm{IC}_{50}$ for LGC-57 compared to LGC-58 and LGC-40 (Figure 2D). Thus, the binding capabilities of tubocurarine on LGC-57 differs substantially from that of its closest family members LGC-58 and LGC-40. Likewise, in the ACC group, LGC-46 and LGC-49 could both be blocked by mecamylamine and strychnine (Figure 2D); LGC-49 could additionally be blocked by tubocurarine and picrotoxin. These dissimilarities again highlight the discrete differences between channels from the same subfamily, which may have similar ligand binding profiles for endogenous ligands. Interestingly, tubocurarine was the most potent blocker for the ACC group channels LGC-46 and LGC-49, whereas this antagonist was the least effective of those tested for the LGC-57 group channels.

We also tested the channels' responses to repeated stimulation by the endogenous primary ligand. We found LGC-40 to be sensitive to repeated stimulation, displaying a significant difference in ratio between the first and second pulse after both $10 \mathrm{~s}$ of and $60 \mathrm{~s}$ of washing intervals (Figure 2C). In contrast, all other channels were capable of fast activation intervals as they did not display any decrease in peak amplitude after repeated stimulation (Figure 2C). Thus, LGC-40 appears to desensitize more than the other channels following activation by its ligand.

\section{LGC-39 is a Novel Polymodal Channel Activated by Cholinergic and Aminergic}

\section{Ligands}

One channel from the LGC-57 subfamily, LGC-39, showed distinct ligand binding properties from the rest of the group. Unlike the other LGC-57 subfamily members, LGC-39 showed relatively little activation by choline. Moreover, while acetylcholine activated LGC- 
39 channels strongly (with an $\mathrm{EC}_{50}$ of $1290 \mu \mathrm{M}$ ), the most potent ligands for LGC-39 were the

Figure 3A-B). In addition, LGC-39 also displayed small currents in response to dopamine (Figure 3A). Like other members of the LGC-57 family, LGC-39 contains the PAR sequence, and LGC-39-expressing oocytes showed reversal potential shifts in response to chloride but not sodium substitution (Figure 3C). Thus, $\lg c$-39 appears to encode a homomeric anionic, polymodal channel, capable of being activated by both aminergic and cholinergic neurotransmitters (Figure 3A-C). be blocked by mecamylamine or strychnine. Surprisingly, strychnine, in the presence of octopamine or without an activating ligand, acted as a partial agonist, inducing a small current with an $\mathrm{EC}_{50}$ of $7.5 \mu \mathrm{M}$ (Figure 3D). These data suggest that the putative endogenous ligands recovery time for the receptor is independent of the activating ligand. 
176 fluorescent reporter lines to characterise the expression pattern of the newly deorphanised

177 LGICs based transcriptional reporter transgenes in which the upstream promoter of the $\lg c$

178 gene drives the expression of a fluorescent protein. We then identified transgene-expression

179 based upon location, morphology and known marker lines. Using such a transcriptional reporter, we observed expression of $l g c-39$ in a range of interneurons and motor neurons,

181 including the AVA premotor interneurons (Figure 4E). In addition to receiving extensive cholinergic input, these neurons are the major synaptic target for the only octopaminergic neurons, the RICs, suggesting that LGC-39 may be exposed to both octopamine and acetylcholine in vivo (Figure 4F) and may be involved in both cholinergic and octopaminergic synaptic transmission. For the rest of the LGC-57 group, we also observed primarily neuronal expression, with little overlap observed in the neurons expressing reporters for $\lg c-40$, $\lg c-49$, $\lg c-57$ and $\lg c-58$ (Figure 4A-D). This was particularly striking in motorneurons, with $\lg c-40$ expressed in many pharyngeal neurons (M2, M3, MC, MI, I2), $\operatorname{lgc}-49$ in sensory neurons, including posterior sensory neurons such as ALN and PLN, $\lg c-57$ in the A-class and B-class motorneurons of the ventral cord, and $\operatorname{lgc}-58$ in the egg-laying motorneurons (VCs; $l g c-57$ was also observed in a subset of VCs) This suggests that these channels are likely to exist primarily as homomers in vivo and function in distinct target neurons.

We also used reporters to analyse the expression pattern of several still-orphan LGICs, including $\lg c-42$, $\lg c-47, \lg c-48, \lg c-43$ and $\lg c-45$, as well as the previously deorphanised ACh-gated channel lgc-46 (Takayanagi-Kiya et al, 2016) (Figure S2A). These reporters also showed diverse and distinct patterns of expression, primarily in neurons. For example, $\operatorname{lgc}-46$ 
199

200

201

202

203

204

205

206

207

208

209

210

211

212

213

214

215

channels were also expressed specifically in neurons; for $\operatorname{lgc}-47$ this expression was unusually broad, encompassing sensory, motor, and interneurons, while $\operatorname{lgc}-48$ was expressed only in a single pair of ADL chemosensory neurons. Interestingly, two the orphan channels, $\lg c-43$, and $\operatorname{lgc}-45$, which lack a PAR sequence and may thus encode cationic channels, did not appear to be expressed in neuronal tissue, but instead in the hypodermis (Figure S2A). Together, these data suggest that these channels play various roles in, and outside, the nervous system.

\section{Excitatory and Inhibitory ACh Receptors are Co-expressed in Many Neurons}

Our expression analysis indicated that many of the inhibitory ACh receptors newly deorphanised in this study are expressed in neurons previously shown to also express excitatory $\mathrm{ACh}$ receptors. These results imply that the role for acetylcholine as an inhibitory neurotransmitter may be greater than previously assumed, and that acetylcholine contributes to both inhibitory and excitatory events in many neurons. To determine the extent to which excitatory and inhibitory receptors for the same neurotransmitter are expressed in individual neural classes, we made use of single cell RNAseq dataset from C. elegans neurons (Taylor et $a l, 2021)$. We first generated a complete list of ionotropic receptors for each of the three classical neurotransmitters ACh, GABA and glutamate (Table S1). Since missing receptors would have the potential to bias predictions, we predicted the ligand and ion selectivity of orphan channels based upon homology with characterised channels and the presence or absence of a PAR motif in the M2-3 intracellular loop (see Methods).

both inhibitory and excitatory receptors for the same neurotransmitter. This was particularly notable for acetylcholine, with over $60 \%$ of neuron classes expressing both excitatory and inhibitory ACh receptors. In contrast, GABA receptors were more biased toward inhibition, 
with over $40 \%$ of neuron classes only expressing inhibitory GABA receptors (Figure 5A, S3).

225 To make generalised predictions of synaptic polarity, we summed expression of inhibitory and excitatory receptors for each neurotransmitter in each neuron class and assigned synapse

227 polarity based on the most prevalent receptors in each neuron class, assuming that all receptors

228 in a cell are present equally at all synapses (Figure 5C, S4). This analysis suggested that the majority of ACh and Glu synapses are excitatory and most GABA synapses inhibitory, though this varied significantly for individual connections (Figure 5B).

\section{Determining Synaptic Localisation of LGICs}

We reasoned that the single cell RNAseq dataset (Taylor et al, 2021) might also be

useful for predicting the intracellular localization of cholinergic LGICs, as presynaptic receptors would be predicted to be expressed in cholinergic neurons while postsynaptic receptors should be expressed in neurons receiving cholinergic input. To assess the correlation between the number of cholinergic synapses a neuron makes ('ACh out'), or receives ('ACh in'), with the expression level of cholinergic LGICs, we produced two heatmaps representing either ACh out (Figure 6A), or in (Figure 6B), correlated to transcript level (Figure S5). This analysis identified some receptors, such as $\operatorname{lgc}-49$, that were primarily expressed in neurons

241 that receive a high number of cholinergic synapses (VD, DD; Figure 6C), consistent with a postsynaptic receptor. Other channels were primarily expressed in neurons that make many

243 cholinergic synapses, such as acc-1 in SMB and SAB (Figure 6D), consistent with presynaptic

244 localization. Others, such as $\operatorname{lgc}-40$ showed little correlation to cholinergic in- or out-degree

245 (Figure 6E), suggesting extrasynaptic localization and function or as for $\operatorname{lgc}-46$ a similar 246 correlation between in or out degree (Figure 6F). 

intracellular M3/4 loop and the function of the resulting chimeric protein was verified in

Xenopus oocytes (Figure S6A). We observed a clear difference in the localisation patterns for these channels. LGC-39::GFP was localised in distinct punctate structures both in the nerve with the positive correlation between $\lg c$-39 expression and cholinergic in-degree. In contrast,

LGC-40::GFP appeared to have a diffuse nerve ring and touch receptor neuron GFP signal

(Figure 7B), with cell bodies often being visible, while LGC-57::GFP was found to be very

LCG-58 showed a small but significant positive correlation with cholinergic out-decree, this extrasynaptic and/or presynyaptic.

\section{Discussion}

\section{A Novel Family of Cholinergic LGICs}

This study highlights the diversity among cholinergic LGICs in C. elegans. Nematodes have previously been shown to contain excitatory LGICs related to nicotinic receptors, as well as inhibitory acetylcholine-gated chloride channels of the ACC family. Here we describe a new subfamily of cholinergic LGICs, four of which form homomeric acetylcholine-gated anion 
gated preferentially by choline, the metabolite of acetylcholine which is abundant at

274 cholinergic synapses.

These results add to the already-extensive catalogue of acetylcholine-gated channels in the expansion and importance of cholinergic transmission in nematodes. These newly deorphanised channels display subtle variations in their ability to bind ligands and antagonists, which translates into physiologically relevant differences that may increase the fine tuning in the control of neuronal transmission and contribute to complex neuronal signalling within a relatively minimal neuronal network. Interestingly, the electrophysiologically similar channels in vivo. When tagged with a fluorescent protein, these three channels also showed a diffuse pattern of localisation within the neuron, suggesting that in contrast to ACC group channels such as LGC-46 (Takayanagi-Kiya et al, 2016), they may not be synaptically localised. This suggests a possible distinct extrasynaptic role for choline acting via these channels in the modulation of the nervous system.

Our results raise the intriguing possibility that choline itself may function as a neuromodulator. The idea that choline could activate cholinergic receptors independently from dose-dependently either block or activate nicotinic channels (Purohit \& Grosman, 2006). However, the importance of choline activation to the in vivo functions of these channels has remained unclear. Here we have identified three cholinergic receptors, LGC-40, LGC-57 and 
degree for choline over acetylcholine. Previous reports show that in mammalian

stabilising the binding of acetylcholine over the binding to choline (Bruhova \& Auerbach, channels in vivo.

\section{A Polymodal LGIC Activated by Both Aminergic and Cholinergic Ligands}

expression pattern of $\operatorname{lgc}-39$ suggests that the channel might be exposed to all these ligands in

AVA neurons also receive some input from tyraminergic and dopaminergic neurons, transmitters which we also found can activate LGC-39. Interestingly, in contrast to the cholinegated channels LGC-40, LGC-57, and LGC-58, we observe clear punctate localisation of LGCaminergic neurotransmission.

The concept of a truly polymodal receptor has not before been investigated in great 
currents (Hoerbelt et al, 2015), while D-serine can function as an allosteric modulator of

325 NMDA receptor activity (Wolosker \& Balu, 2020). Based on their capability to achieve dose-

326 dependent activation, both amines and acetylcholine appear to be true ligands of LGC-39, most

327 likely binding the ligand-binding domain. Understanding the mechanisms by which these multiple neurotransmitters can activate LGC-39 and potentially affect different behavioural outputs will be of interest in future studies.

\section{Functional Insights into The $C$. elegans Connectome}

With increasing molecular and physiological characterisation of neurotransmitter receptors in C. elegans, it is becoming feasible to predict the functionality of synapses more accurately in the C.elegans connectome. In this study we used the expression pattern of newly and previously deorphanised LGICs for the three classical neurotransmitters, ACh, glutamate and GABA, to predict the polarity of synapses in the C. elegans connectome. By assigning synapse polarity based on the relative expression levels of anionic and cationic receptors, we have provisionally predicted the sign of chemical synapses involving classical neurotransmitters. Although similar attempts have been made in the past to assign polarity to C. elegans synapses (Fenyves et al, 2020), these predictions were based upon incomplete or

341 incorrect ligand assignment for many LGICs. These revised predictions correlate well with

342 experimental data for many well-characterised circuits, such as the excitatory connections 343 between the ASH nociceptors and the AVA interneurons, as well as between the AVAs and 344 the VA and DA motorneurons (Mellem et al, 2002; Piggott et al, 2011). Predicted inhibitory 345 connections between AVB and AVA interneurons also correspond with empirical data on the

346 locomotor circuit (Kawano et al, 2011; Qi et al, 2012). Other predictions were counterintuitive;

347 for example, inhibitory connections were predicted between the AVDs and AVAs, two 
interneuron pairs thought to be co-ordinately active during reverse locomotion (Faumont et al,

A surprising outcome of the expression analysis was the high frequency with which

neurons expressed cationic and anionic receptors for the same neurotransmitter. This was

especially prevalent for acetylcholine; our analysis indicated that $60 \%$ of neuron classes

for GABA. One explanation for this apparent paradox is that excitatory and inhibitory receptors

might be differentially localised in neurons, with some found extrasynaptically and others

enriched in at synapses. Various C.elegans LGICs are known to act in regions other than the

(Jobson et al, 2015), while $\operatorname{lgc}-46$ appears be localised to the pre-synapse as an autoreceptor (Takayanagi-Kiya et al, 2016). The choline-sensitive channels from the LGC-57 group

likewise appear to be extrasynaptic in their localization (Figure 7). In addition, postsynaptic sites might themselves contain a mixture of excitatory and inhibitory receptors, which could experience.

\section{Materials and Methods}


373 Unless otherwise specified, C. elegans worms were cultured on NGM agar plates with OP50

374 (Stiernagle, 2006). A full list of strains used in this study can be found in table S1.

375

376 Xenopus laevis oocytes

377 Defolliculated Xenopus laevis oocytes were obtained from EcoCyte Bioscience (Dortmund,

378 Germany) and in ND96 (in mM: $96 \mathrm{NaCl}, 1 \mathrm{MgCl}_{2}, 5 \mathrm{HEPES}, 1.8 \mathrm{CaCl}_{2}, 2 \mathrm{KCl}$ ) solution at

$37916^{\circ} \mathrm{C}$ for $3-7$ days.

\section{Molecular biology}

381 Unless otherwise specified, cDNA sequences of $C$. elegans genes were cloned from wildtype 382 N2 worm cDNA (generated by reverse transcription PCR from total worm RNA using Q5 383 polymerase (New England Biosciences)). Where multiple isoforms are present isoform a was 384 used. LGC-39 cDNA was generated by gene synthesis (ThermoFischer). For expression in 385 Xenopus oocytes, ion channel cDNA sequences were cloned into the KSM vector downstream of a T3 promoter and between Xenopus $\beta$-globin 5' and 3'UTR regions using the HiFi assembly protocol (New England Biosciences). C. elegans expression constructs were also generated using the $\mathrm{HiFi}$ assembly protocol (New England Biosciences) into the pDESTR4R3II backbone. C. elegans gDNA sequences were cloned from wildtype N2 gDNA and expression verified by the addition of GFP or mKate 2 introduced on the same plasmid after an intercistronic splice site (SL2 site). Unless otherwise specified promoter sequences consist of approximately $2 \mathrm{~kb}$ of gDNA upstream of the start codon.

\section{CRISPR/CAS9-mediated gene manipulation}

395 Endogenous tagging of the M3/4 cytosolic loop of C. elegans LGIC proteins with GFP was carried out either using the SapTrap protocol (Schwartz \& Jorgensen, 2016; Dickinson et al, 
2018) for $\lg c-39(\operatorname{lj} 121)$, or by SunyBiotech (Fuzhou, China) for $\lg c-57(\operatorname{syb3536)}, \lg c$ -

58(syb3562), and $\operatorname{lgc}-40(\operatorname{syb3594).}$

399

400 RNA synthesis and microinjection

401

cRNA was synthesised in vitro using the T3 mMessage mMachine transcription kit according

402 to manufacturer's protocol to include a 5' cap (ThermoFischer Scientific). Prior to injection

403 RNA was purified using the GeneJET RNA purification kit (Thermo Fischer Scientific). Size sorted and defolliculated Xenopus oocytes (Ecocyte) were placed individually into 96-well

$500 \mathrm{ng} / \mu \mathrm{L}$, with a 1:1 ratio of the components. Injected oocytes were incubated at $16^{\circ} \mathrm{C}$ in

ND96 until the day of recording, typically between 3-6 days post injection.

409

\section{Two-Electrode Voltage Clamp (TEVC) recording and data analysis}

411 Two-electrode voltage clamp recordings were carried out using either the Robocyte2 system 412 or a manual set up with an OC-725D amplifier (Multi Channel Systems GmbH). Glass 413 electrodes with a resistance ranging from 0.7-2 M $\Omega$ were pulled on a P1000 Micropipette Puller 414 (Sutter). Electrodes contained $\mathrm{AgCl}$ wires and backfilled with a $1.5 \mathrm{M} \mathrm{KCl}$ and $1 \mathrm{M}$ acetic mixture. Unless otherwise stated, oocytes were clamped at $-60 \mathrm{mV}$. Continuous recordings at $500 \mathrm{~Hz}$ were taken during application of a panel of agonists or antagonists. Data was recorded

417 using the RoboCyte2 control software, or with WinWCP for manual recordings, and filtered at $10 \mathrm{~Hz}$.

Dose response protocols used 10s agonist application pulses with 60s of wash in ND96 between 
422 Peak current for each dose was normalised to the oocyte maximum current using a custom-

423 built python script (Morud et al, 2021). Normalised data was imported into Graphpad (Prism)

424 and fitted to either a three or four parameter nonlinear Hill equation to obtain the highest degree

425 of fit and calculate the $\mathrm{EC}_{50}$. Antagonist dose responses and ion selectivity recordings were

426 carried out using the $\mathrm{EC}_{50}$ concentration of the primary agonist. Antagonist dose response

427 protocols used 10s agonist + antagonist windows, with 60s of ND96 washes between doses.

428 The agonist concentrations remained constant. Antagonist $\mathrm{IC}_{50}$ values were calculated using a

429 second custom built python script (Morud et al, 2021). Normalised data was imported into

430 Graphpad (Prism) and fitted to either a three or four parameter nonlinear Hill equation to obtain

431 the highest degree of fit and calculate the $\mathrm{IC}_{50}$.

432

433 Ion selectivity was detected using a voltage ramp protocol from $-80 \mathrm{mV}$ to $+60 \mathrm{mV}(20 \mathrm{mV} / \mathrm{s})$

434 in the presence of the primary agonist in three different solutions: ND96, NMDG ( $\mathrm{Na}^{+}$free)

435 and $\mathrm{Na}$ Gluconate (low $\mathrm{Cl}$ ) solutions. Data was normalised to max current and $\Delta \mathrm{E}_{\mathrm{Rev}}$ was

436 calculated using a custom-built python script (Morud et al, 2021). The resulting individual

437 values or mean, SD and $\mathrm{n}$ for each construct was imported in GraphPad for further plotting and

438 statistical analysis. Statistically significant differences in $\Delta \mathrm{E}_{\mathrm{Rev}}$ were calculated in GraphPad

439 using a two-way ANOVA with Tukey's correction for multiple comparisons. A representative

440 normalised trace for each construct was also generated in Graphpad.

\section{Confocal and Cell ID}

443 Worms were prepared and immobilised with $75 \mathrm{mM} \mathrm{NaAzide}$ in M9 and mounted onto 2\%

444 agarose in M9 pads. Image stacks were acquired with a $63 \mathrm{x}$ water immersion lens on a Leica

445 SP8 or STED or using a 40x oil immersion objective on a Zeiss LSM780. Collapsed z-stack

446 images were generated in Fiji/Image J. Neurons expressing fluorescent reporters were 
447 identified by cell shape, position and crossing with the multicolour reference worm NeuroPAL

448 (Yemini et al, 2020).

\section{Synaptic polarity prediction}

451 Inhibitory and excitatory chemical synapse prediction for ACh, Glu and GABA synapses were

452 made based upon expression levels of appropriate LGICs in postsynaptic cells. Chemical and

453 electrical connectome data was obtained from Wormweb (http://wormweb.org/details.html),

454 LGIC expression data was taken from the Cengen project using threshold level 4 (Taylor et al,

455 2021), ligand and selectivity for each channel was based upon this work, previous work and

456 predictions, a full list can be found in Table 1. Binary expression of LGICs for each

457 neurotransmitter in each neuron class were made based upon expression and characterised in

458 four groups: only excitatory, only inhibitory, both excitatory and inhibitory or none. These

459 binary values were used to make the binary expression heatmap. Overall polarity of a synapse

460 was calculated by summing the expression of all inhibitory and all excitatory LGICs for a given

461 neurotransmitter in each cell class. The sum inhibitory was then taken from the sum of

462 excitatory expression, resulting in an overall positive or negative signed expression in each

463 neuron class for each neurotransmitter. The ratio of these sums was also calculated to indicate

464 the strength of polarity. It was assumed that each LGIC in each neuron class is present equally

465 in all synapses, therefore each incoming connection could be assigned a polarity based upon

466 its receptor expression for that neurotransmitter. The resulting network with polarity was

467 imported into cystoscope (Shannon et al, 2003) for plotting and further analysis. Analysis

468 scripts can be found on GitHub at hiris25/Worm-Connectome-Polarity.

469

470 Expression and cholinergic synapse analysis 
471 The total number of cholinergic input or output synapses was calculated for each neuronal class

472 by summing the number of presynapses for each cell that received a synapse from an ACh-

473 producing neuron (receiving synapses), or the total number of post-synapses an ACh-producing

474 neuron class makes (sending synapses). Acetylcholine producing cells were described by

475 (Pereira et al, 2015), the assumption was that all synapses made by an ACh-producing cell also

476 release ACh, even when this cell co-transmits another neurotransmitter. Synapse number for

477 each neuron was taken from (White, 1986). Expression data was obtained from (Taylor et al,

478 2020) using a stringent threshold of 4 . Neuronal classes were sorted by ACh in or out degree

479 and the expression of each gene was mapped using a heatmap with an upper threshold of 500.

480 Correlation between expression level and ACh in, or out, degree was mapped using relplot in

481 python's seaborn package, confidence intervals were placed at $68 \%$, corresponding to the

482 standard error of the estimate.

483

484 Data analysis

485 Unless otherwise specified data was imported into GraphPad for further analysis and plotting.

486 Unless otherwise specified, one-way ANOVA was used to calculate significant differences, 487 using the Bonferroni (food leaving \& dispersal) or Tukey's (food leaving time course, 488 chemotaxis) correction for multiple comparisons. $*=\mathrm{P}<0.05, * *=\mathrm{P}<0.005, * * *=\mathrm{P}<0.001$, $* * * * \mathrm{P}<0.0001$

490

\section{Data Availability}

492 Python scripts can be found at on GitHub at hiris25/TEVC-analysis-scripts and hiris25/Worm493 Connectome-Polarity. Aggregated data used for analysing TEVC data are available upon request from the Lead Contact. Further information and requests for C. elegans strains and 
plasmids is to be sent to and will be fulfilled by the Lead Contact William $\mathrm{R}$ Schafer,

\section{Acknowledgements}

499 The authors gratefully acknowledge Denise Walker and Lidia Ripoll Sanchez for help with

500 cloning design, help with generating and maintaining strains, and other past and present

501 members of the Schafer lab for helpful discussions. We would like to acknowledge the Centre

502 for Cellular Imaging at the University of Gothenburg, Sweden, and the National Microscopy

503 Infrastructure, NMI (NMI01125), for providing imaging facilities. This work was supported

504 by grants from the Medical Research Council (MC-A023-5PB91), the Wellcome Trust

505 (WT103784MA), National Institute of Health (W.R.S.), the Swedish Research council

506 (VR2017-00236), Knut and Alice Wallenberg foundation (KAW2019,0293), Bollan stipend,

507 Lundgrenska stiftelserna and Magnus Bergvalls Stiftelse (J.M.).

508

\section{Author Contributions}

510 I.H., J.M. and W.R.S. designed the experiments. I.H., J.M., performed experiments and 511 analysed data. I.H., J.M. and W.R.S. wrote the manuscript and all authors read and critically

512 revised the manuscript to its final form.

513

\section{Conflicts of Interest}

515 The authors declare no competing interests.

\section{References}

518 Alkema M \& Horvitz B (1999) Determining the roles of octopamine and CREB in worm 
520 Bondarenko V, Targowska-Duda KM, Jozwiak K, Tang P \& Arias HR (2014) Molecular interactions between mecamylamine enantiomers and the transmembrane domain of the human $\alpha 4 \beta 2$ nicotinic receptor. Biochemistry 53

Brams M, Pandya A, Kuzmin D, van Elk R, Krijnen L, Yakel JL, Tsetlin V, Smit AB \& Ulens C (2011) A structural and mutagenic blueprint for molecular recognition of strychnine and d-tubocurarine by different Cys-loop receptors. PLoS Biol 9

Bruhova I \& Auerbach A (2017) Molecular recognition at cholinergic synapses: acetylcholine versus choline. J Physiol 595

Cully DF, Paress PS, Liu KK, Schaeffer JM \& Arena JP (1996) Identification of a Drosophila melanogaster glutamate-gated chloride channel sensitive to the antiparasitic agent avermectin. J Biol Chem

Cully DF, Vassilatis DK, Liu KK, Paress PS, Van Der Ploeg LHT, Schaeffer JM \& Arena JP (1994) Cloning of an avermectin-sensitive glutamate-gated chloride channel from Caenorhabditis elegans. Nature

Dickinson D, Slabodnick M, Chen A \& Goldstein B (2018) SapTrap assembly of repair templates for Cas9-triggered homologous recombination with a self-excising cassette. microPublication Biol 2018

Faumont S, Lindsay TH \& Lockery SR (2012) Neuronal microcircuits for decision making in

$$
\text { C. elegans. Curr Opin Neurobiol } 22 \text { doi:10.1016/j.conb.2012.05.005 [PREPRINT] }
$$

Fenyves BG, Szilágyi GS, Vassy Z, Sőti C \& Csermely P (2020) Synaptic polarity and sign- 
544 Gisselmann G, Pusch H, Hovemann BT \& Hatt H (2002) Two cDNAS coding for histamine-

545 gated ion channels in D. melanogaster. Nat Neurosci

546 Hobert O (2013) The neuronal genome of Caenorhabditis elegans. WormBook

547 doi:10.1895/wormbook.1.161.1 [PREPRINT]

548 Hoerbelt P, Lindsley TA \& Fleck MW (2015) Dopamine directly modulates GABAA

$549 \quad$ receptors. J Neurosci 35

550 Jobson MA, Valdez CM, Gardner J, Garcia LR, Jorgensen EM \& Beg AA (2015) Spillover

551 transmission is mediated by the excitatory GABA receptor LGC-35 in C. elegans. $J$

$552 \quad$ Neurosci

553 Jones AK \& Sattelle DB (2008) The cys-loop ligand-gated ion channel gene superfamily of

554 the nematode, Caenorhabditis elegans. Invertebr Neurosci 8: 41-47

555 Kawano T, Po MD, Gao S, Leung G, Ryu WS \& Zhen M (2011) An imbalancing act: Gap

556 junctions reduce the backward motor circuit activity to bias C. elegans for forward

$557 \quad$ locomotion. Neuron 72

558 Kondo M, Nakamura Y, Ishida Y, Yamada T \& Shimada S (2014) The 5-HT3A receptor is

$559 \quad$ essential for fear extinction. Learn Mem 21

560 Liu P, Chen B, Mailler R \& Wang ZW (2017) Antidromic-rectifying gap junctions amplify

561 chemical transmission at functionally mixed electrical-chemical synapses. Nat Commun

562 Lombaert N, Hennes M, Gilissen S, Schevenels G, Aerts L, Vanlaer R, Geenen L, Van

563 Eeckhaut A, Smolders I, Nys J, et al (2018) 5-HTR 2A and 5-HTR 3A but not 5-HTR

564 1A antagonism impairs the cross-modal reactivation of deprived visual cortex in

$565 \quad$ adulthood. Mol Brain 11

566 Malinow R \& Malenka RC (2002) AMPA receptor trafficking and synaptic plasticity. Annu 
Mellem JE, Brockie PJ, Zheng Y, Madsen DM \& Maricq A V. (2002) Decoding of receptor for learning. Curr Biol

Pereira L, Kratsios P, Serrano-Saiz E, Sheftel H, Mayo AE, Hall DH, White JG, LeBoeuf B,

Piggott BJ, Liu J, Feng Z, Wescott SA \& Xu XZS (2011) The neural circuits and synaptic mechanisms underlying motor initiation in C. elegans. Cell 147

Pirri JK, McPherson AD, Donnelly JL, Francis MM \& Alkema MJ (2009) A Tyramine-Gated

\section{Escape Response. Neuron 62}

582 Purohit Y \& Grosman C (2006) Block of muscle nicotinic receptors by choline suggests that the activation and desensitization gates act as distinct molecular entities. J Gen Physiol

Putrenko I, Zakikhani M \& Dent JA (2005) A family of acetylcholine-gated chloride channel subunits in Caenorhabditis elegans. J Biol Chem 280

Qi YB, Garren EJ, Shu X, Tsien RY \& Jin Y (2012) Photo-inducible cell ablation in Caenorhabditis elegans using the genetically encoded singlet oxygen generating protein miniSOG. Proc Natl Acad Sci U S A 109

Ranganathan R, Cannon SC \& Horvitz HR (2000) MOD-1 is a serotonin-gated chloride channel that modulates locomotory behaviour in C. elegans. Nature 408

Ringstad N, Abe N \& Horvitz HR (2009) Ligand-gated chloride channels are receptors for biogenic amines in C. Elegans. Science (80- ) 325 
594 Schwartz ML \& Jorgensen EM (2016) SapTrap, a toolkit for high-throughput CRISPR/Cas9

595 gene modification in Caenorhabditis elegans. Genetics 202

596 Shannon P, Markiel A, Ozier O, Baliga NS, Wang JT, Ramage D, Amin N, Schwikowski B

597 \& Ideker T (2003) Cytoscape: A software Environment for integrated models of

$598 \quad$ biomolecular interaction networks. Genome Res 13

599 Stiernagle T (2006) Maintenance of C. elegans. WormBook doi:10.1895/wormbook.1.101.1

600 [PREPRINT]

601

Takayanagi-Kiya S, Zhou K \& Jin Y (2016) Release-dependent feedback inhibition by a

602 presynaptically localized ligand-gated anion channel. Elife

603

Taylor SR, Santpere G, Weinreb A, Barrett A, Reilly MB, Xu C, Varol E, Oikonomou P,

Taylor SR, Santpere G, Weinreb A, Barrett A, Reilly MB, Xu C, Varol E, Oikonomou P, system. Cell 184

White JG. (1986) The structure of the nervous system of the nematode Caenorhabditis

Wolosker H \& Balu DT (2020) d-Serine as the gatekeeper of NMDA receptor activity: implications for the pharmacologic management of anxiety disorders. Transl Psychiatry

614 Wotring VE, Miller TS \& Weiss DS (2003) Mutations at the GABA receptor selectivity filter: A possible role for effective charges. $J$ Physiol 548 DEG-3/DES-2 receptor: A nicotinic acetylcholine receptor that mutates to cause neuronal degeneration. Mol Cell Neurosci 17 
619

620

621

622

623

624

625

626

627

628

629

630

631

632

633

634

635

636

637

638

639

640

641

642

643

Yemini E, Lin A, Nejatbakhsh A, Varol E, Sun R, Mena GE, Samuel ADT, Paninski L, Venkatachalam V \& Hobert O (2020) NeuroPAL: A Multicolor Atlas for Whole-Brain Neuronal Identification in C. elegans. Cell

\section{Figure Legends}

Figure 1. Deorphanisation of Cholinergic Ligand Gated Ion Channels. (A) Continuous current traces of Xenopus oocytes expressing LGC-57, LGC-58a, LGC-40, and LGC-49, oocytes were perfused for 10s with a panel of ligands each at $1 \mathrm{mM}$ : ACh (acetylcholine), Ch (choline), Bet (betaine), TA (tyramine), DA (dopamine), 5-HT (serotonin), OA (octopamine), GA (GABA), GLU (glutamate), His (histamine), MA (melatonin). (B) Phylogenetic tree of a subgroup of Cys-loop ligand gated ion channels in C.elegans, groups of interest are highlighted by colour: 'ACC' group of acetylcholine gated channels (red), orphan 'cationic diverse' group (yellow), orphan LGC-57 group (green). Gene names in bold are deorphanised in this study. (C) Dose response curves for LGC-57, LGC-58a, LGC-40 and LGC-49 in response to their major ligand(s). Current is normalised by I/Imax for each oocyte, for LGC-49 current is raw un-normalised current. Error bars represent SEM of 5-14 oocytes. Curves are fitted with a fourparameter variable slope, inserts show $\mathrm{EC}_{50}$ in $\mu \mathrm{M}$ for each ligand.

Figure 2. Ion Selectivity and Antagonistic Characterisation of Cholinergic LGICs. (A) Representative current voltage plots of newly de-organised channels in ND96, Na gluconate or NMDG solutions. Current was normalised by subtraction of leak current (in absence of activating ligand) and to the peak current for each oocyte. (B) Box plot of $\Delta \mathrm{E}_{\text {rev }}$ of NMDG and Na Gluconate vs. ND96 in oocytes expressing LGC-57, LGC-58a, LGC-40, LGC-39, LGC46, and LGC-49, $\mathrm{E}_{\mathrm{rev}}$ was calculated in the presence of the major agonist of each channel and 
644 leak subtracted. N=6-11 oocytes. (C) Current ratio of oocytes expressing LGC-57, LGC-58,

645 LGC-46, LGC-49 or LGC-39 undergoing repeated agonist application with 10s, 30s and 60s

646 wash intervals. Error bars represent SEM. N=5-9 oocytes per condition. (D) Antagonist

647 application in the presence of ligands using mecamylamine, strychnine, tubocurarine and

648 picrotoxin. Current is normalised by I/Imax for each oocyte, curves are fitted with a three-

649 parameter variable slope, error bars represent SEM of 2-7 oocytes, inserts show $\mathrm{IC}_{50}$ in $\mu \mathrm{M}$ for

650 each antagonist.

651

652

Figure 3. LGC-39 Forms a Polymodal Ligand-Gated Ion Channel. (A) Continuous current

trace of a Xenopus oocyte clamped at $-60 \mathrm{mV}$ expressing LGC-39, perfused during 10s pulses

(glutamate), His (histamine). (B) Dose response curve for LGC-39 in response to ACh, Oct

dose response curves for LGC-39 expressing oocytes activated by either ACh, Oct or no ligand

(E) Three different agonists do not differ in how they influence the ability for LGC-39 to be stimulated with short time intervals after repeated stimulation.

Figure 4. Newly Deorphanised LGICs are Expressed Broadly in the Nervous System. (A- 
and/or genomic sequence of reveals broad neuronal expression of cholinergic channels with

670 little overlap. (F) Schematic depicting a subset of the synaptic connections received by the $\operatorname{lgc}$ -

67139 expressing neuron class, AVA, numbers in brackets show the total number of synapses for

672 each connection.

673

674 Figure 5. Predicting Synapse Polarity Based on LGIC Expression. (A) Bar chart depicting

675 the percentage of total neuron classes expression inhibitory, excitatory, both or no receptors

676 for GABA, glutamate (Glu) and acetylcholine (ACh). (B) Bar chart depicting the percentage

677 of total synapses for a given neurotransmitter that are predicted to be inhibitory, excitatory or

678 have no prediction. (C) Network diagram depicting the polarity of synaptic connections

679 between neuron classes, connection colour shown polarity: teal (inhibitory), pink (excitatory),

680 grey (no prediction). Gap junctions are represented by dashed lines. Line weight represents

681 number of synapses and nodes are coloured by the major neurotransmitter a class release.

682 Diagram made with cytoscape using the EntOpt clustering package. (D) Network diagram 683 depicting the predicted polarity of the locomotion circuit, connection colour shows polarity:

684 teal (inhibitory), pink (excitatory), grey (no prediction). Inserts show the fold magnitude of 685 expression of the major receptor type for each neurotransmitter in each neuron class. Gap 686 junctions are represented by dashed lines. Line weight represents number of synapses and 687 nodes are coloured by the major neurotransmitter a class release. Diagram made with cytoscape.

Figure 6. Correlation of cholinergic synapses with expression pattern of cholinergic ion neuron class. Neurons are sorted by the total number of cholinergic synapses they make (top, 
the total number of cholinergic synapses made (orange) or received (red) by a neuron and

Figure 7. Protein expression pattern of cholinergic ion channels. (A-D) Localisation of endogenously GFP tagged LGC-39, LGC-40, LGC-57 and LGC-58. White arrows highlight areas of interest, represented in higher magnification below.

\section{Expanded View Figure Legends}

Supplementary Table 1: The list shows ligand identity and ion selectivity for LGICs in $C$.

has been noted in the list. representative curves and channel kinetics.

A. Continuous TEVC traces from oocytes clamped at $-60 \mathrm{mV}$ expressing LGC-32, LGC-33, LGC-34, LGC-42, LGC-44, and LGC-48, exposed to 10s of a panel of ligands. B. LGC-47 and ACC-1 do not form a heteromeric channel in Xenopus oocytes. Left: Acetylcholine induced dose response curves for oocytes expressing ACC-1 alone or in combination with LGC-47.

715 Error bars represent SEM of 7-12 oocytes per construct, insert shows EC $_{50}$ values. Right:

716 Continuous TEVC traces from oocytes clamped at $-60 \mathrm{mV}$ expressing LGC-47, ACC-1 or a combination, exposed to 10 s of a panel of ligands. 
A. Expression of fluorescent reporters for still orphan LGCIs. B. Acetylcholine induced dose

response curve, continuous trace, and expression pattern of LGC-46. Error bars represent SEM expression pattern with expression in e.g., AIZ, RIH and AVE. expression of LGICs in white.

The heatmap shows the summed expression level, and ion selectivity, for LGICs separated by transmitter (ACh, glutamate and GABA) and neuron class. Net excitatory channel expression is represented in pink and inhibitory in green.

741 Scatter plots showing gene expression level vs total no. cholinergic synapses received (red) the line fit. 
745 Supplementary Figure 6: GFP tagging of LGICs does not influence channel function.

746 Acetylcholine or choline induced dose response curves for oocytes expressing GFP tagged

747 version of LGC-40, LGC-57 and LGC-58 shows that $\mathrm{EC}_{50}$ values are not influenced by the

748 insertion of the GFP tag. Error bars represent SEM of 7-12 oocytes per construct, insert shows

$749 \quad \mathrm{EC}_{50}$ values.

750

751 
bioRxiv preprint doi: https://doi.org/10.1101/2021.10.06.463318; this version posted October 7, 2021. The copyright holder for this preprint (which was not certified by peer review) is the author/funder, who has granted bioRxiv a license to display the preprint in perpetuity. It is made available under aCC-BY-NC 4.0 International license.

\section{Figures:}

753 Figure 1

A.

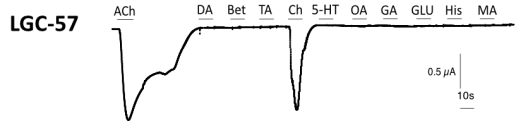

LGC-58a

LGC-40

LGC-49

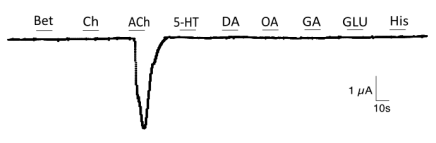

B.

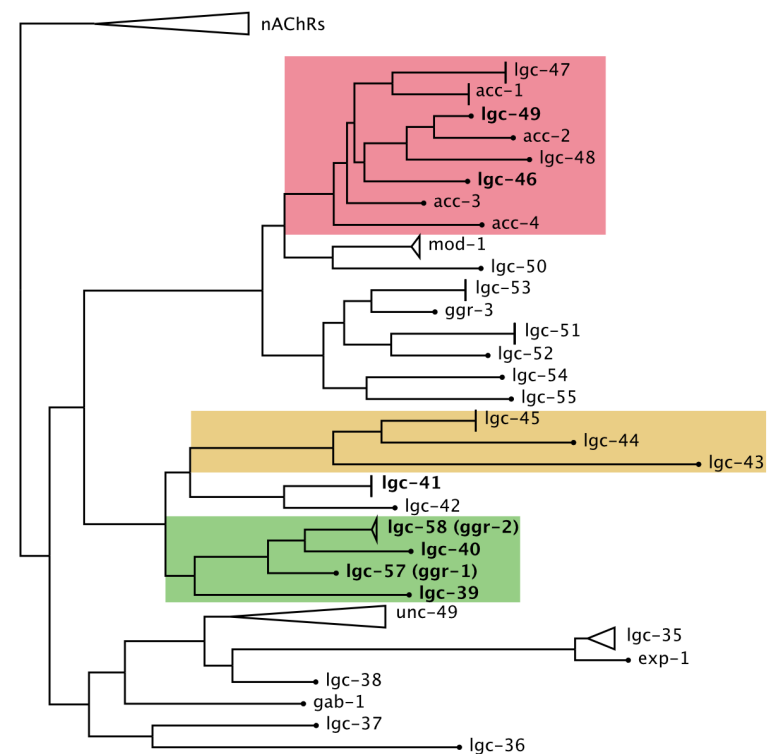

C.

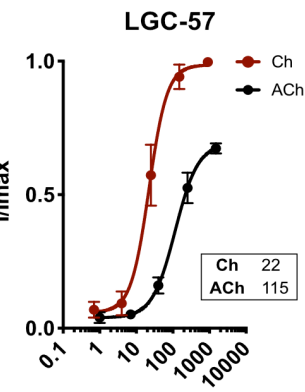

[Agonist] $(\mu \mathrm{M})$

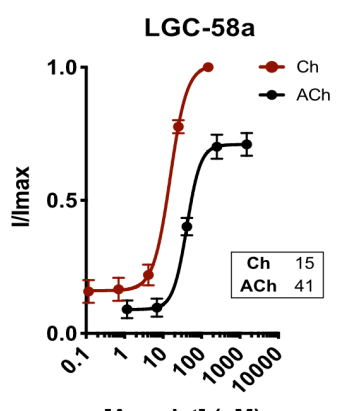

[Agonist] $(\mu \mathrm{M})$
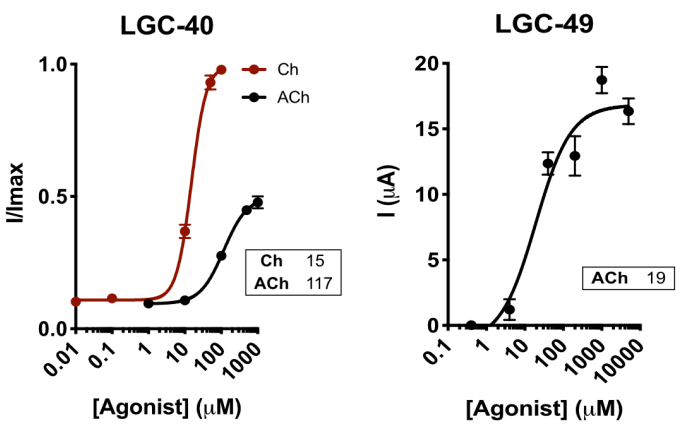
bioRxiv preprint doi: https://doi.org/10.1101/2021.10.06.463318; this version posted October 7, 2021. The copyright holder for this preprint (which was not certified by peer review) is the author/funder, who has granted bioRxiv a license to display the preprint in perpetuity. It is made available under aCC-BY-NC 4.0 International license.

Figure 2

A. LGC-57
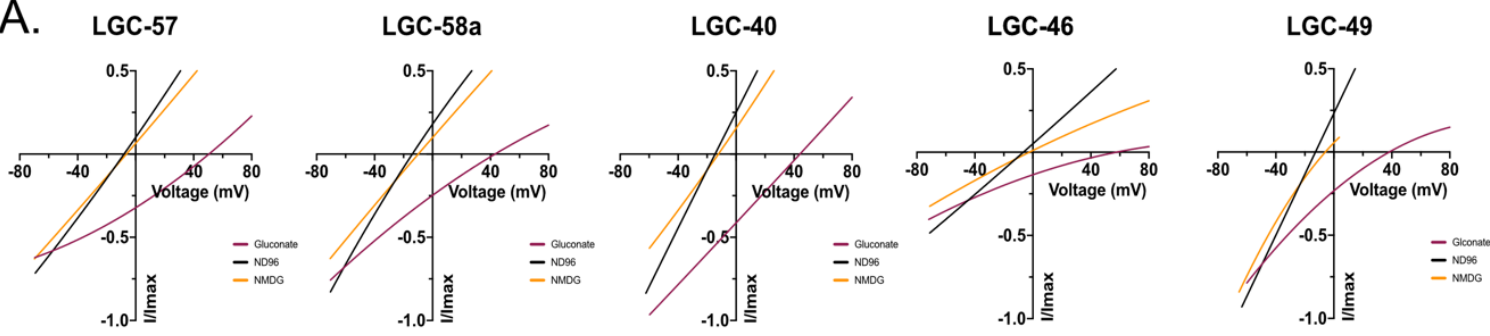

B.

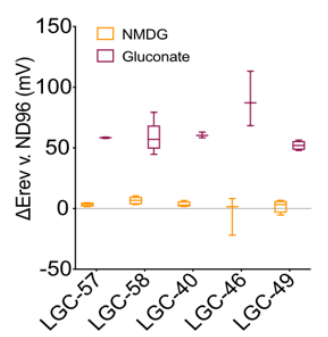

C.

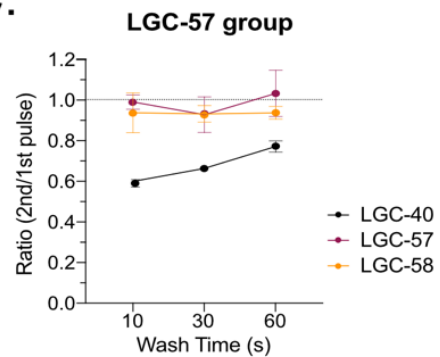

D.

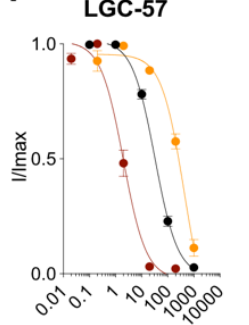

Antagonist $(\mu \mathrm{M})$

- Mecamylamine 32

Strychnine 2.0
LGC-58a

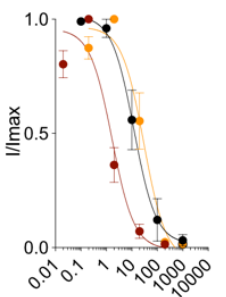

Antagonist ( $\mu \mathrm{M})$

- Mecamylamine 12

Strychnine 1.7
LGC-40

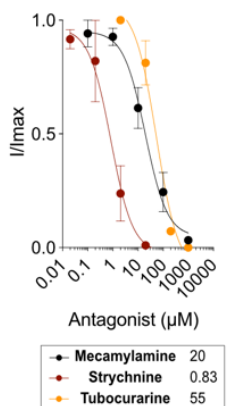

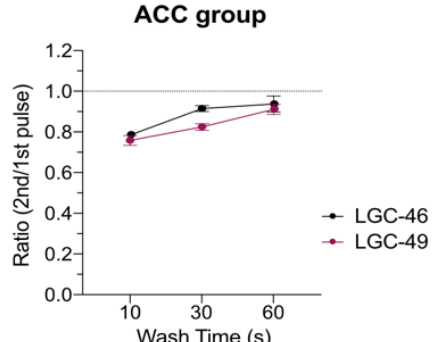

LGC-46

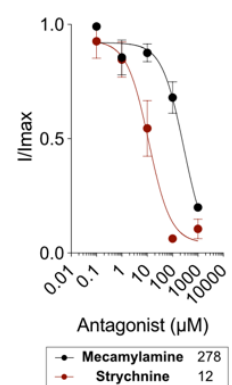

LGC-49

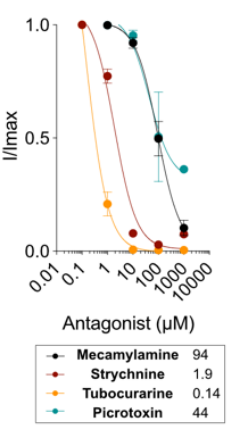


bioRxiv preprint doi: https://doi.org/10.1101/2021.10.06 463318; this version posted October 7, 2021. The copyright holder for this preprint (which was not certified by peer review) is the author/funder, who has granted bioRxiv a license to display the preprint in perpetuity. It is made available under aCC-BY-NC 4.0 International license.

757 Figure 3

A.

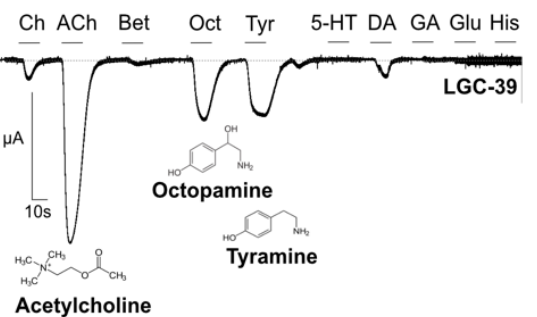

D.

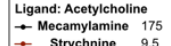 - Strychnine 9.5}

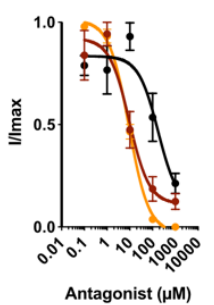

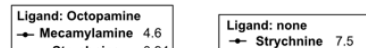

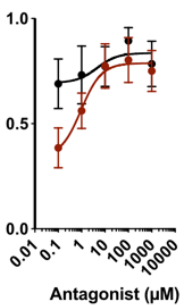

758

759

Figure 4
B.

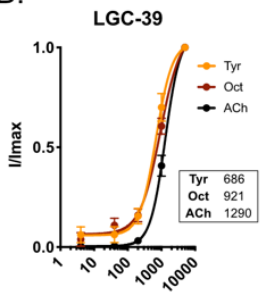

$\log$ [Agonist] (uM)

E.

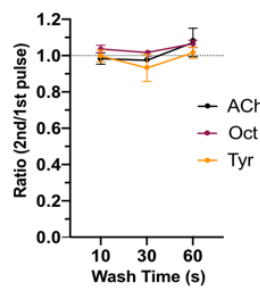

C.
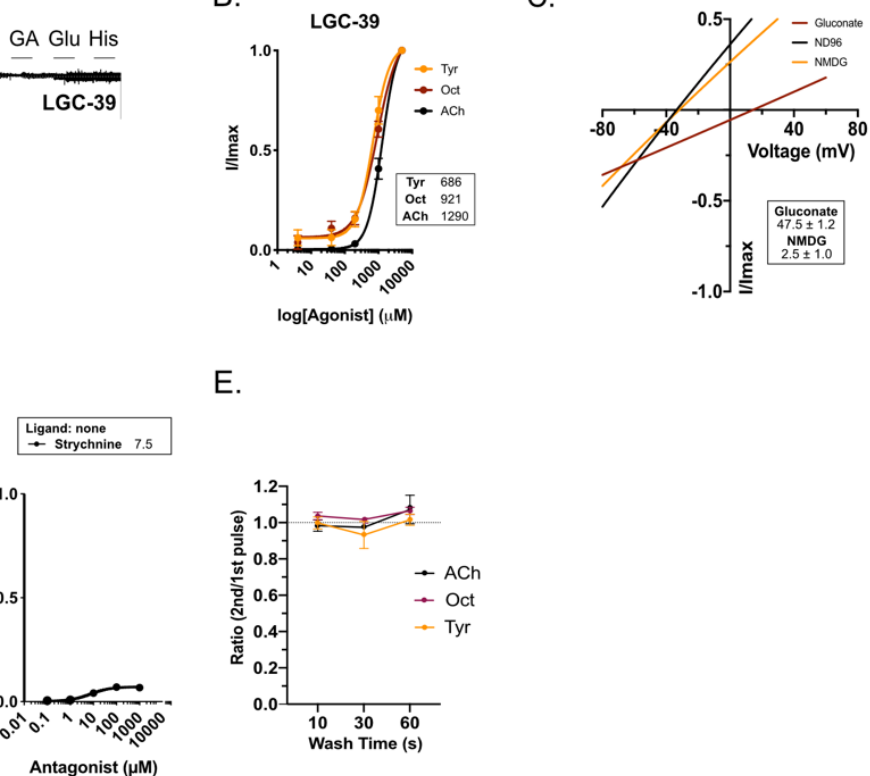

HEAD

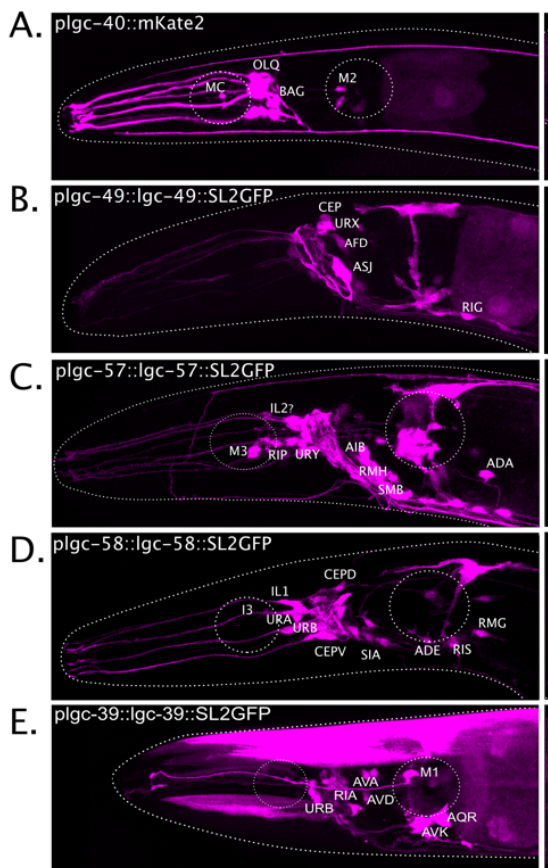

MIDBODY

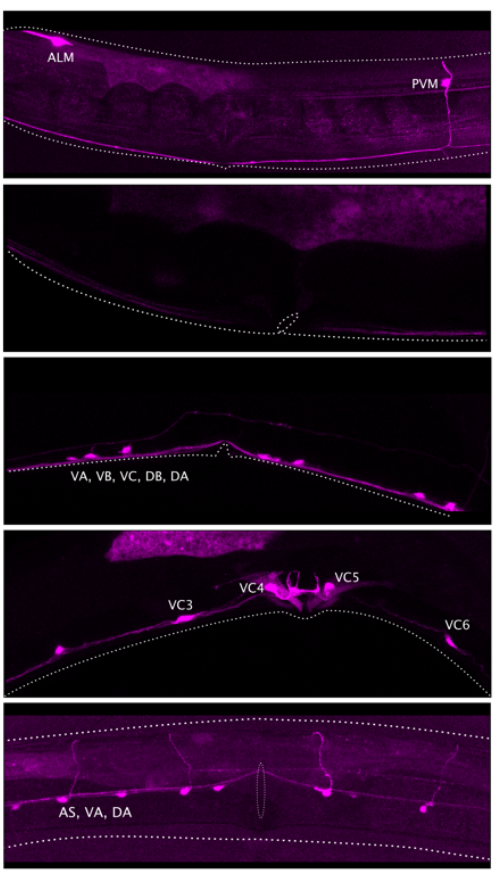

TAIL
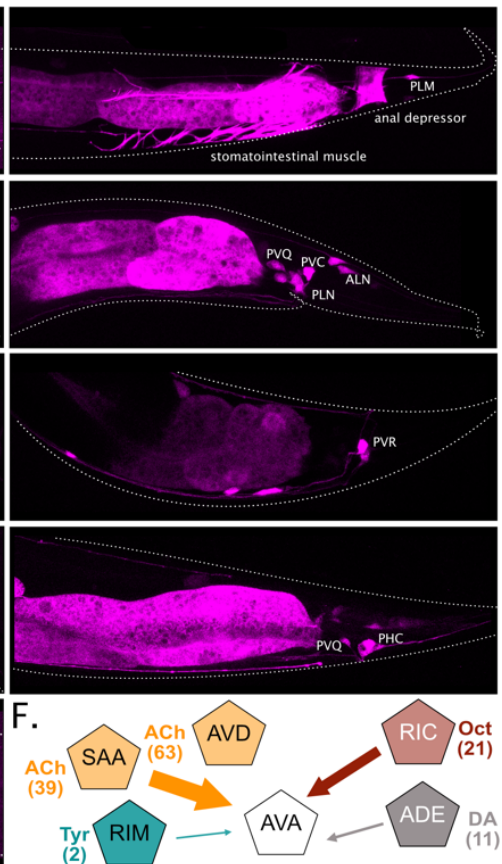
bioRxiv preprint doi: https://doi org/10.1101/2021.10.06 463318; this version posted October 7, 2021. The copyright holder for this preprint (which was not certified by peer review) is the author/funder, who has granted bioRxiv a license to display the preprint in perpetuity. It is made available under aCC-BY-NC 4.0 International license.

Figure 5

A.

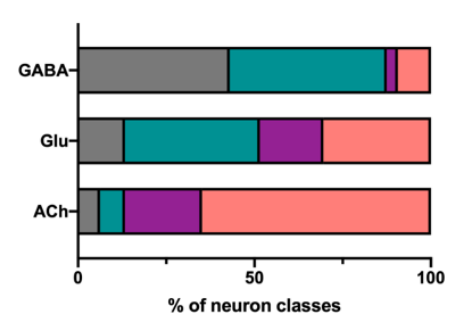

B.

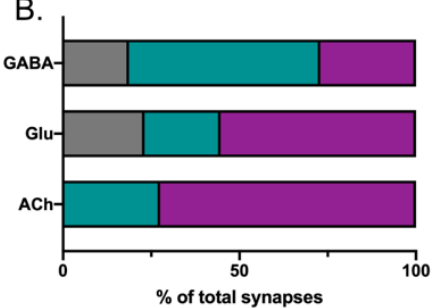

C.

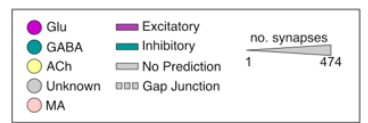

는 Inhibitory

믈tatory

口 Bot

ㅁ No Prediction

믄 Inibitory

- Exctatory

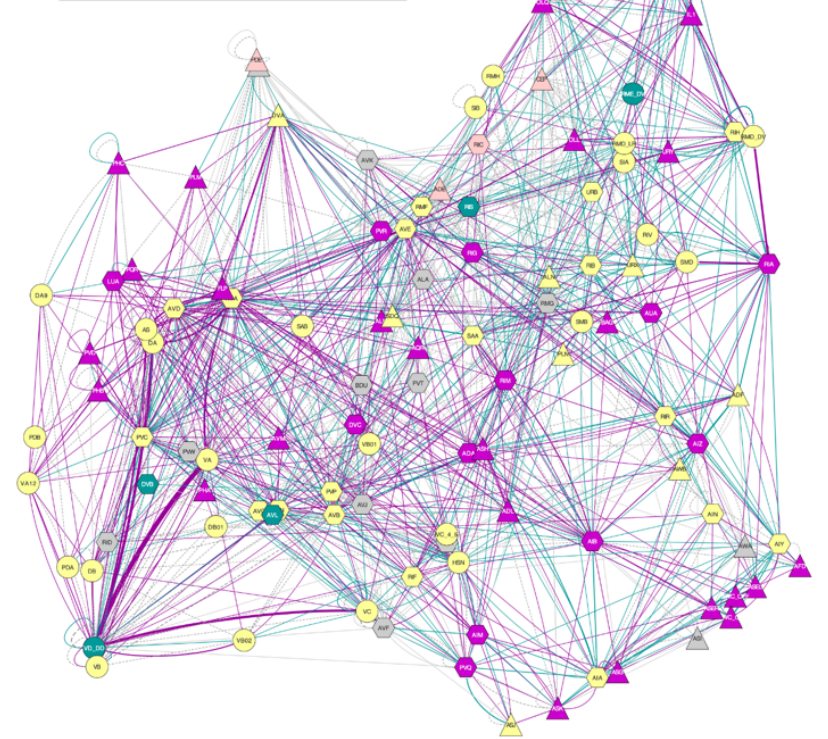

D.

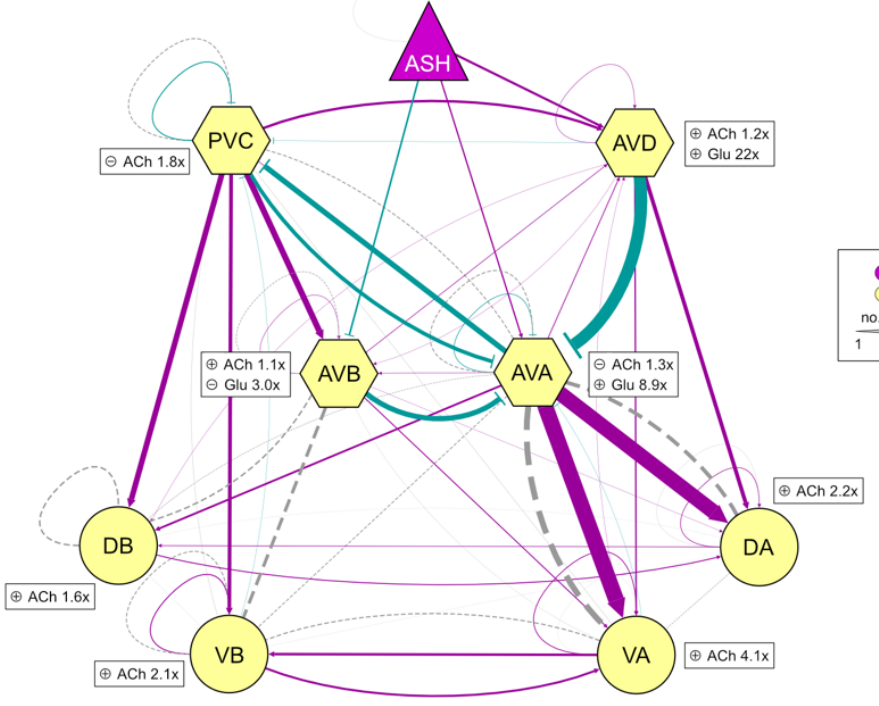

Glu $=$ Excitatory

no. synapses $\square$ No Prediction

FORWARDS

BACKWARDS 


\section{A.}

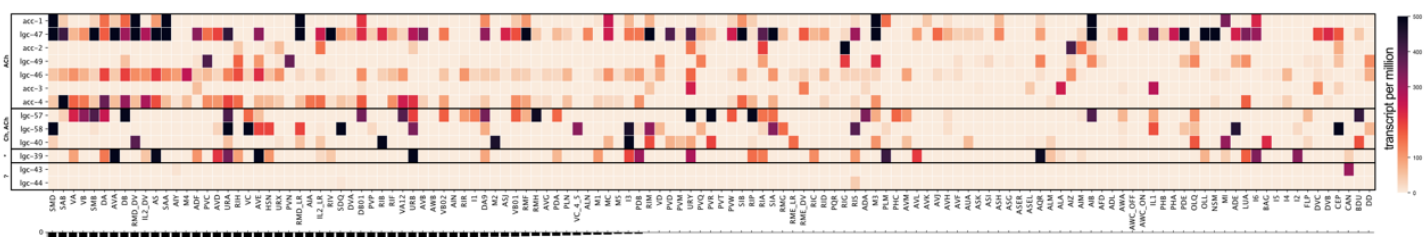

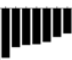

B.

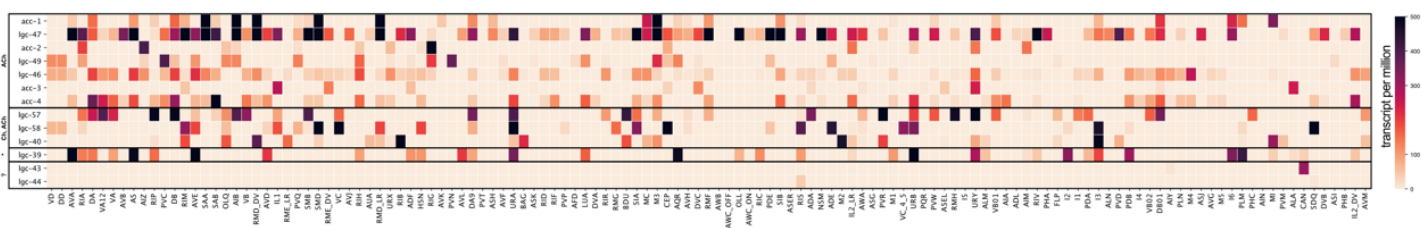
||

C.

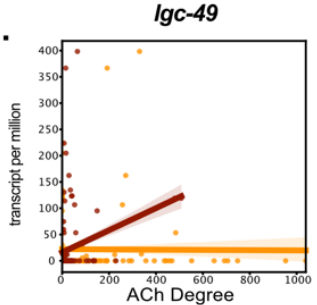

D.

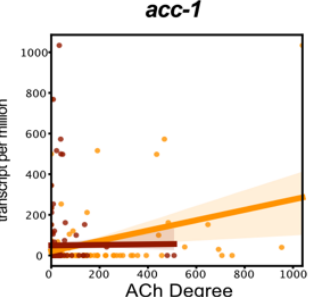

E.

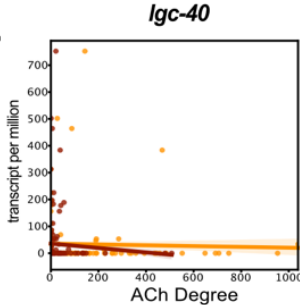

F.

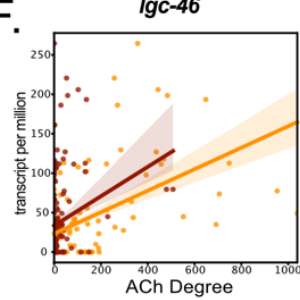


bioRxiv preprint doi: https://doi.org/10.1101/2021.10.06 463318; this version posted October 7, 2021. The copyright holder for this preprint (which was not certified by peer review) is the author/funder, who has granted bioRxiv a license to display the preprint in perpetuity. It is made available under aCC-BY-NC 4.0 International license.

Figure 7

A.
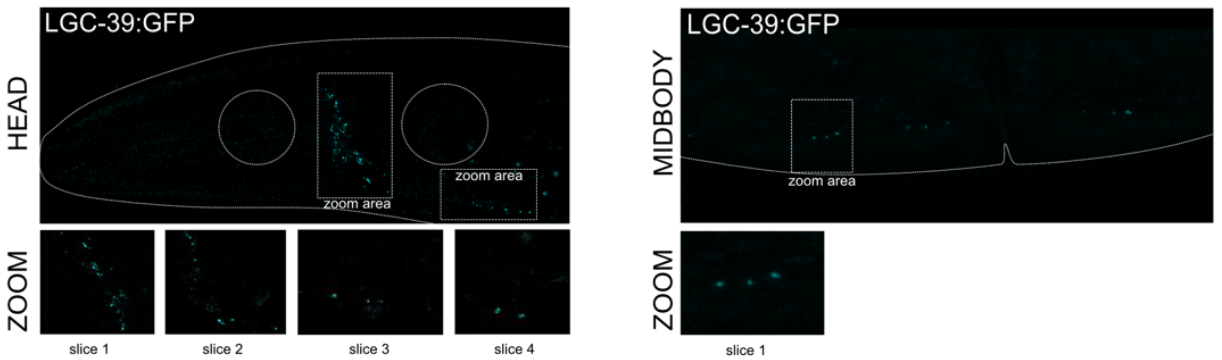

B.
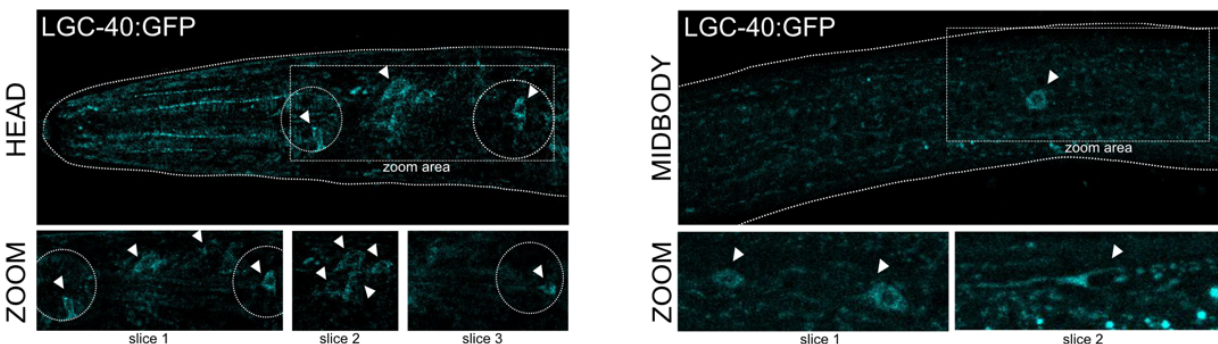

C.
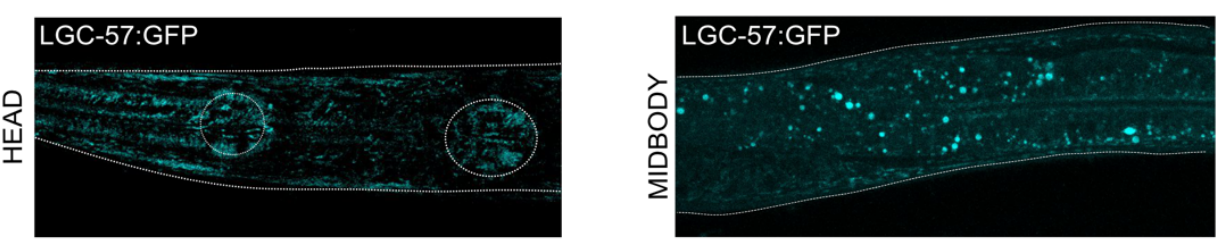

D.
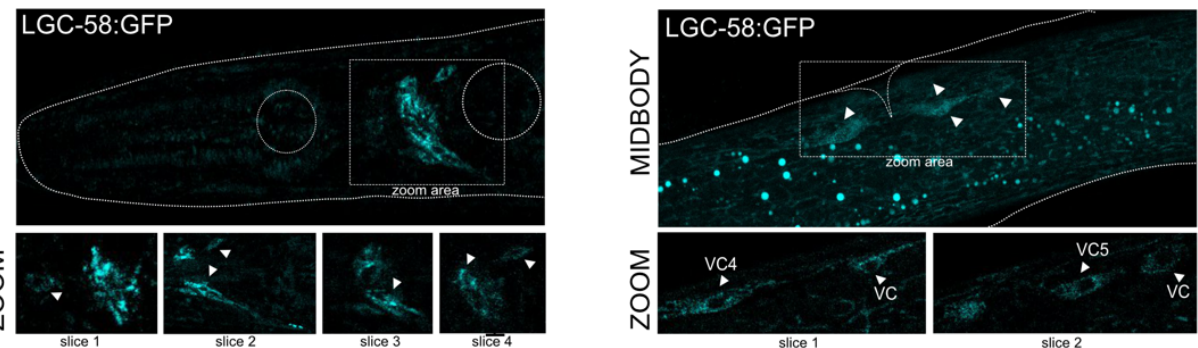